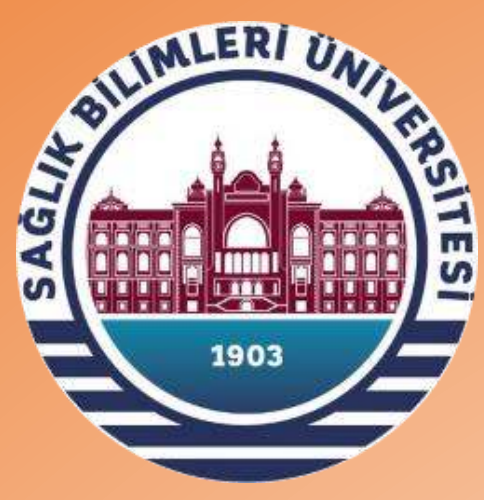

\title{
CARE OF EPIDERMOLYSIS BULLOSA PATIENTS
}

Ayşe Göbekli', Rabiye Güney²

${ }^{1}$ MSN Student, University of Health Sciences, Health Sciences Institute, Nursing

Sciences Main Branch, İstanbul

${ }^{2}$ PhD, RN, University of Health Sciences, Faculty of Nursing, Pediatric Nursing Department, İstanbul

\section{BACKGROUND AND AIMS}

Epidermolysis Bullosa (EB) which is rare and has many subtypes, is a group of genetic diseases characterized by erosion on mucous membranes and eruption (blister formation) occuring after minimal traumas and impaired dermo-epidermal layer integrity of the skin. This study was conducted in order to draw attention to epidermolysis bullosa disease, to reveal the nursing care that is evidenced by current studies and to exemplify the new studies in nursing field.

\section{METHOD}

While this systematic review is being studied, ULAKBIM (Turkish Article Database), Google Academic, PubMed, and Science Direct databases were scanned using the words of "Epidermolysis Bullosa, rare diseases and nursing care". This review was established with 22 articles as a result of the searches done and inclusion criteria applied. Important results presented here.

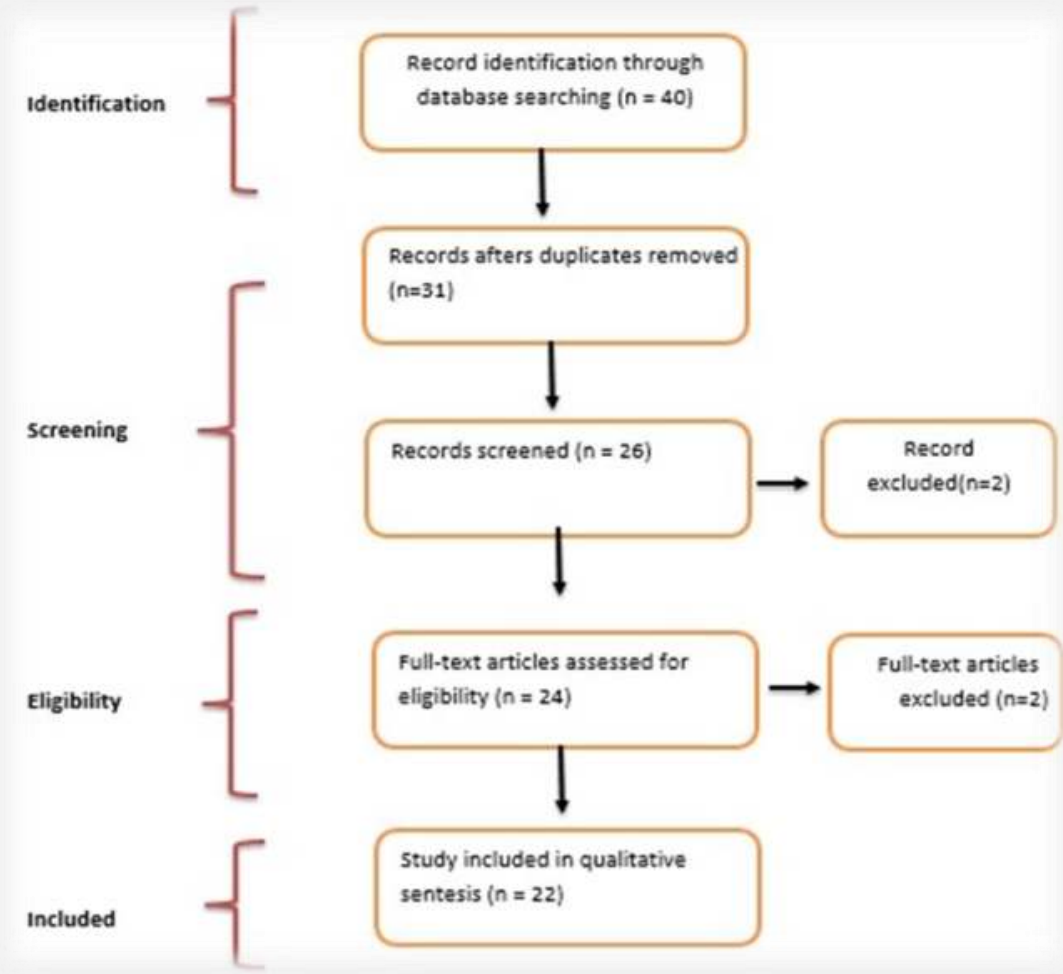

\section{RESULTS}

The common syptom in all types of epidermolysis bullosa was the occurance of recurrent blistering and erosions following minimal trauma.

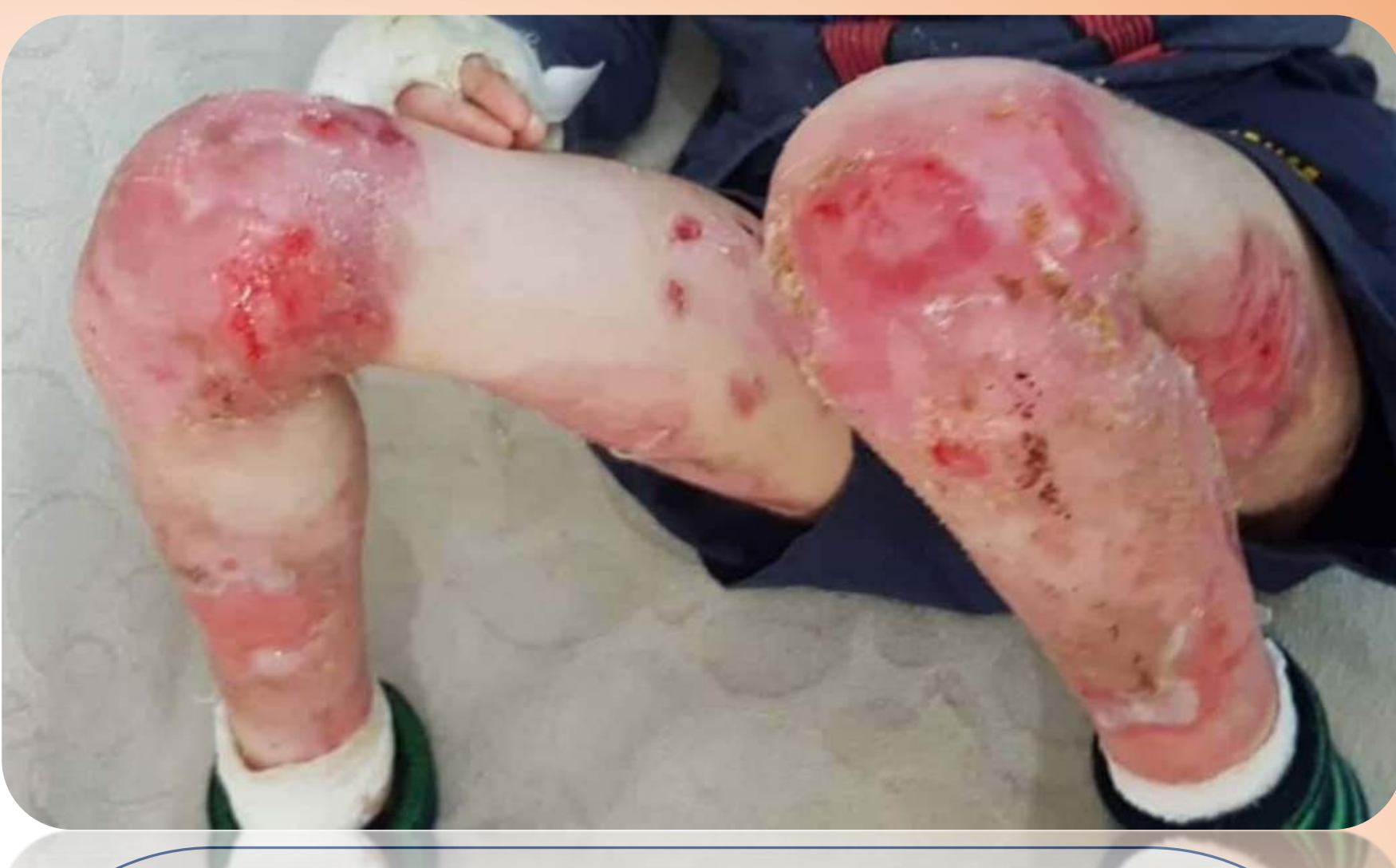

Incidence and prevelence rates of EB was unclear. According to some reports, it was seen in one out of eight-nine million people. Although there was no spesific treatment, the aims of the treatment were following; firstly to avoid trauma, prevent secondary infections, accelerate wound healing and provide nutritional support.

Over the years, many drugs and dressings have been evaluated in the wound care of EB disease. In Hon's study (2005), dystrophic EB wounds were covered with honeyimpregnated bandages and wound healing was observed in 15 weeks. In a study conducted by Lynne et al. (2018), Allevyn Gentle Border Lite bandage has been found to be more economical and healthy than Mepitel-Mepilex and in patients with this bandage increase in dressing changing time.

It should be remembered that taking into account the psychosocial aspect of the disease, including the care and treatment of the disease requires a multidisciplinary approach. Tabolli at al.(2010) in their study, found that the burden of caregivers of children with epidermolysis bullosa is similar to the burden of caregivers of cancer patients.

In Australia in 2012, a home nursing care system was set up and its effectiveness was assessed to help patients with severe EB disease and their families by Stevens et al (2016). The findings drew attention to the comfort of families when receiving professional nursing services and showed that home care nursing is needed for children and their families with EB.

\section{CONCLUSION}

Since EB is a chronic disease, it is very important to treat affected children and their families with a multidisciplinary team and utilize a wide range of nursing approaches. Studies for improving treatment and quality of life of patients with EB are still ongoing. Qualitative researches on the experiences of children with EB and their families are needed. More studies are needed to investigate and reveal the socio-economic conditions and financial resources of families with EB.

\section{REFERENCES}

- Hon J. Using honey to heal a chronic wound in a patient with epidermolysis bullosa. British Journal of Nursing 2005;14(Sup5), S4-S12.

- Lynne, V., Burns, L., Handsaker, J., \& Murdoch, J. M. (2018). Epidermolysis bullosa: management complexities for paediatric patients. British Journal of Nursing, 27(Sup12), S20-S25.

- Tabolli, S., Pagliarello, C., Uras, C., Pietro, C. D., Zambruno, G., Castiglia, D., ... \& Abeni, D. (2010). Family burden in epidermolysis bullosa is high independent of disease type/subtype. Acta dermato-venereologica, 90(6), 607-611.

Stevens, L. J., McKenna, S., Marty, J., Cowin, A. J., \& Kopecki, Z. (2016). Understanding the outcomes of a home nursing programme for patients with epidermolysis bullosa: an Australian perspective. International wound journal, 13(5), 863-869. 